Erratum

\title{
Fractal geometry of critical Potts clusters
}

E.M. Rauch ${ }^{\mathrm{a}}$

MIT Computer Science and Artificial Intelligence Laboratory, 32 Vassar St., Cambridge, MA 02139, USA

Eur. Phys. J. B 34, 497 (2003)

Received 2 January 2005

Published online 11 February 2005 - C EDP Sciences, Società Italiana di Fisica, Springer-Verlag 2005

An error occurred in the author's list of the article by J. Asikainen et al., Eur. Phys. J. B 34, 497 (2003): The name of the co-author E.M. Rauch was misspelled (it read Rausch instead of "Rauch"). Therefore the correct reference of the article is:

Fractal geometry of critical Potts clusters

J. Asikainen, A. Aharony, B.B. Mandelbrot, E.M. Rauch, J.-P. Hovi, Eur. Phys. J. B 34, 497 (2003).

\footnotetext{
a Present address: Department of Ecology and Evolutionary Biology, Princeton University, Princeton, NJ 08544-1003, USA e-mail: rauch@ai.mit.edu
} 